\title{
Microstructural signatures of dislocation avalanches in a high-entropy alloy
}

\author{
Q. Rizzardi, ${ }^{1}$ P. M. Derlet, ${ }^{2}$ and R. Maaß $\circledast^{1,3, *}$ \\ ${ }^{1}$ Department of Materials Science and Engineering and Frederick Seitz Materials Research Laboratory, \\ University of Illinois at Urbana-Champaign (UIUC), Urbana, Illinois 61801, USA \\ ${ }^{2}$ Condensed Matter Theory Group, Paul Scherrer Institute, Villigen, PSI 5232, Switzerland \\ ${ }^{3}$ Federal Institute of Materials Research and Testing (BAM), Unter den Eichen 87, 12205 Berlin, Germany
}

(Received 22 December 2020; accepted 10 March 2021; published 15 April 2021)

\begin{abstract}
Here, we trace in situ the slip-line formation and morphological signature of dislocation avalanches in a highentropy alloy with the aim of revealing their microstructural degree of localization. Correlating the intermittent microplastic events with their corresponding slip-line patterns allows defining two main event types, one of which is linked to the formation of new slip lines, whereas the other one involves reactivation of already existing slip lines. The formation of new slip lines reveals statistically larger and faster avalanches. The opposite tendency is seen for avalanches involving reactivation of already existing slip lines. The combination of both these types of events represents the highest degree of spatial avalanche delocalization that spans the entire sample, forming a group of events that determine the truncation length scale of the truncated power-law scaling. These observations link the statistics of dislocation avalanches to a microstructural observable.
\end{abstract}

DOI: 10.1103/PhysRevMaterials.5.043604

\section{INTRODUCTION}

Fluctuations in plasticity have for a long time been a topic mainly dedicated to material systems that exhibit directly observable stress-strain discontinuities during deformation. This includes the well-known cases of dynamic strain aging (DSA) in, for example, AlMg alloys [1] and mild steels [2], or inhomogeneous deformation of metallic glasses (MGs) [3]. In these classes of materials, the underlying physics that governs the unstable plastic response are markedly different, involving a dynamic interaction between solutes and dislocations in DSA, whereas a competition between athermally-imparted shear disordering and thermally-activated relaxation governs the serrated flow behavior of MGs [4]. These mechanistic origins are a deviation from the norm of generally observed smooth plastic flow in bulk materials and are not only of fundamental academic interest. In fact, the associated strain localization can constitute severe challenges in metal forming and may cause significant mechanical property degradation. In the more unusual case of single crystalline metals considered here, only a few unique experimental observations have been reported that indicate the existence of a more general spatiotemporal intermittent stress-strain behavior [5].

Indeed, based on the seminal work by Orowan [6] and others, our typical approach to describe crystal plasticity involves phenomenological functions that capture a continuous flow response and that rely on average quantities such as a mean dislocation or obstacle spacing, a mean grain size, or a mean obstacle strength. Such homogenization schemes are fundamentally based on Gaussian-like statistics with well-defined mean values and stand in stark contrast to a few selected bulk

\footnotetext{
*robert.maass@bam.de
}

deformation studies that report scale-free deformation [7-9]. The signature of scale-free and thus non-Gaussian dislocation activity has been revealed with acoustic-emission sensing on bulk hexagonal closest packed (hcp) crystals $[10,11]$ and a plethora of recent small-scale deformation experiments across numerous material systems [12-21]. One of the dominant findings was that a lot of experimental data showed agreement with analytical statistical physics frameworks, notably a pinning-depinning model for avalanches near the depinning transition [22]. In such an approach, one relies on long-range elastic coupling, a static pinning field, and a locally varying critical stress. Once this stress is reached, elastic coupling allows for avalanche phenomena, which here is considered to be a collective dislocation event. The ability of the model to capture the probability $P$ of plastic event sizes $S$ with a power law (PL) or truncated PL (TPL), where a similar numerical value of the exponent $\alpha$ was found for many materials, motivated the idea of a scale-free and universal property of plasticity [23].

Since dislocation-based plasticity involves the continuous evolution of a three-dimensional dislocation network, where the pinning points consequently change in terms of number, spacing, and strength, a jamming-unjamming transition was proposed instead of a depinning transition in a static pinning field $[24,25]$. Based on this view, the statistical signature of intermittent collective dislocation activity also follows a PL distribution, but now the size-scaling exponent does exhibit a range of nontrivial values. The prevalence of nontrivial scaling exponents has also been reported experimentally [25], and given the various parameters (lattice type, crystal orientation, drive rate, strain-hardening rate) that seemingly affect the numerical value of $\alpha$, care must be taken when confirming models via experimental results. Specifically, before determining $\alpha$ from a given intermittent microplastic deformation 
curve, the critical drive rate below which $\alpha$ becomes independent of rate needs to be determined [26]. This can be in the subnanometer per second regime, which is much lower than typically considered. Despite the increasing complexity regarding the scaling behavior of plastic fluctuations, this recent development points toward nonuniversal intermittent microplasticity [14,25,27-29], which would be in good agreement with the classical material physics approach that relies on microstructural-specific processes and scales.

The aforementioned results were based on stress-strain (or force-displacement) measurements of deforming crystals, where most of the experimental work originated from small-scale plasticity. This means that statistical datasets are produced from a series of deformation curves, and there is no further microstructural information available. In response to this shortcoming, we have earlier proposed to regard a dislocation avalanche as a microstructural dynamical object [5], which entails some degree of microstructural signature that we now consider.

Specifically, we aim here at establishing a direct connection between a given event size, its probability of occurring, and its degree of spatial localization. Indeed, so far, the literature has only considered postmortem slip-line patterns, which means that it remains unknown if any given event size is related to slip confined to one slip plane or if the activation of numerous separated slip planes occurred during the avalanche. Similarly, when examining the spatiotemporal velocity profiles of the dislocation avalanche size statistics $[12,27,30]$, the simultaneous occurrence of spatially separated slip would lead to faster velocities, implying nothing else than that the scaling between event size and velocity is solely due to avalanche delocalization. While the degree of spatial localization related to a given event size remains unknown, fundamental work on the emerging slip-step morphologies after deformation has been done [31,32]. Here, self-affine slip-step statistics have been reported for plastically deformed crystals over a large strain regime [33]. Using atomic force microscopy, PL scaling of surface step heights was found for both single crystals [34] and polycrystals [35] with no strong dependence on plastic strain. As such, scale-free signatures of plasticity and therefore underlying stochastic processes that have long-range spatial correlations are revealed with a variety of experimental methods (acoustic emission, stress-strain curves, and measurement of surface steps).

To deepen our understanding of the spatial localization of collective dislocation events, that is dislocation avalanches, we will here pursue in situ electron microscopy experiments with the aim of establishing a 1:1 relationship between a given event and its slip-line morphology. This approach is motivated by our desire to understand if certain parts of the event-size distribution are statistically favoring a particular slip morphology and therefore spatial localization. In the view of our earlier results demonstrating nontrivial and nonuniversal scaling exponents [12-14,25,27,33], it furthermore became of interest what statistical scaling particular types of slip morphologies, if they are definable, may have. These considerations emerged out of our observation that postmortem visualized slip patterns across different types of single crystals can be dramatically different while exhibiting statistical scaling that was practically identical.
This paper details uniaxial microcrystal compression experiments on a single crystalline high-entropy alloy (HEA) that are conducted inside a scanning electron microscope (SEM). The choice of an HEA was based on the high probability of sufficiently large slip events [13] such that slip lines could be resolved inside the SEM. Pausing the quasistatic straining after each detected slip event, images were acquired that subsequently could be analyzed with respect to their differences. This methodology allowed identifying two main groups of slip morphology, involving either a reactivation of already activated slip planes or the formation of new slip lines. Dislocation avalanches proceeding on already activated slip planes are more frequently observed than the activation of new slip planes. These two groups of avalanches contribute differently to the avalanche size distribution and therefore overall scaling exponent, where events on newly activated slip lines lower the exponent, and events of reactivation type contribute to an increase of the exponent. It is further observed that a specific slip morphology involving the simultaneous activation of multiple slip planes exclusively contributes to the extreme value tail of the slip-size distribution and therefore the truncation length scale of the distribution. As such, a high degree of spatial avalanche delocalization that essentially occurs through the entire sample volume defines the largest events that statistically are also the fastest.

\section{EXPERIMENTAL METHODS}

We conducted uniaxial compression tests on a single crystalline $\mathrm{Al}_{0.3} \mathrm{CoCrFeNi} \mathrm{HEA}$. This alloy is a solid solution single-phase face-centered cubic crystal [36] and was prepared in the form of $2 \mu \mathrm{m}$ diameter microcrystals with an aspect ratio of nominally 3 and a loading axis along the $\langle 124\rangle$ axis (single-slip orientation with a Schmid factor of 0.486). This orientation favors glide on the (111)[101] slip system. More than 40 microcrystals were prepared via annual focused ion-beam milling using an FEI Helios 600i DualBeam station. The same dual beam station was used for in situ microcompression experiments by integrating a Bruker-Hysitron Picoindenter PI85. Straining was done in displacement control (i.e., strain controlled for a given total length), at a displacement rate of $6 \mathrm{~nm} \mathrm{~s}^{-1}$ (i.e., a strain rate of $10^{-3} \mathrm{~s}^{-1}$ ), for a total displacement of $150 \mathrm{~nm}$ [i.e., a total plastic strain of 0.025 , see also Fig. 1(a)] per loading. Subsequently, the sample was unloaded and reloaded until another plastic strain increment of 0.025 was reached. It is noted that this displacement rate is well below the critical rate that would affect the slip-size statistics [33]. Following this load-unloading procedure, every microcrystal was deformed to a total strain of $\sim 0.25$, which amounts to $\sim 1500 \mathrm{~nm}$ in displacement. SEM imaging of the slip-line morphology was done before and after slip events, whenever it was possible to pause the test without the occurrence of another slip event. During imaging, the samples were kept under the quasistatic load that was applied just before halting the experiment. To properly image the slip-line morphology, the magnification was changed, but no sample-stage movement occurred. The aim was thus to record the slip-line morphologies of the entire sample before and after an event [Fig. 1(b)]. Whenever slip proceeded in a rapid succession of events, it became impossible to pause the experiment and to 

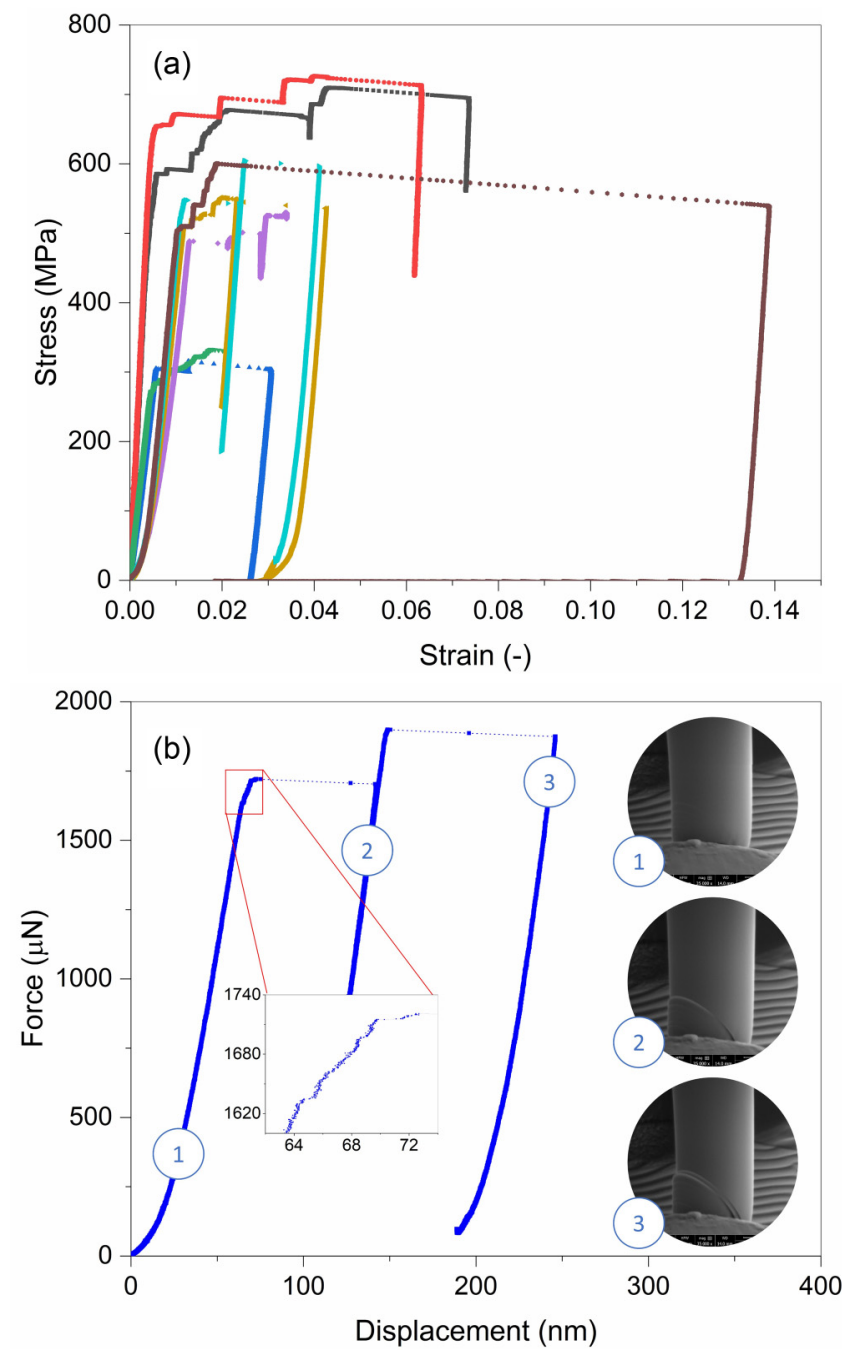

FIG. 1. (a) Typical stress-strain curves obtained from the in situ compression experiments on the high-entropy alloy (HEA) microcrystals. Each sudden displacement jump corresponds to one resolvable dislocation avalanche that may range between a few tenths of a nanometer to $>1 \mu \mathrm{m}$. (b) Image capture process during the experiment. At the start of each experiment, an initial picture is taken; after each recorded mechanical event, the experiment is stopped, and a new picture is taken. This picture represents the "after" image associated with that event, as well as the "before" image for the next, future event. Inset: detail of force-depth curve showing a few small precursor events that could not always be caught during in situ experimentation.

image the slip-line morphology between each individual event because the data-plotting frequency of the real-time monitor during testing is limited to $60 \mathrm{~Hz}$.

The recorded mechanical data (force-displacement) was analyzed to extract the magnitude of axial displacement bursts (event or "avalanche size"). Since each event is the manifestation of a finite number of Burgers vectors that are deposited onto the crystal surface, a simple geometrical factor based on Schmid's law determines the shear slip-size magnitude $d_{s}$ from the axial event size. For a measured axial displacement $d_{v}$ and an angle $\theta$ between the normal of the slip plane and the axially applied stress, the shear displacement is $d_{s}=$ $d_{v} / \sin \theta$. Here, $\theta \approx 51^{\circ}$, and so $d_{s} \approx 1.29 \times d_{v}$. However, for the purpose of our statistical analysis, this geometrical factor is irrelevant, and we will adhere to the axial displacement values as event sizes. We use the same semi-automated routine as in Refs. [12,27] to detect displacement jumps. Finite impulse response filtering was used to reduce the noise of the data. The obtained event sizes are subsequently analyzed statistically using the maximum likelihood estimation (MLE) approach [37] and the POWERLAW Python package [38], with the goal to determine the distribution function that best describes the experimental data.

\section{RESULTS AND DISCUSSION}

Figure 1(a) displays a set of stress-strain curves recorded during in situ SEM testing. Each of these curves corresponds to one loading sequence, and together, they show the variability in both microplastic yielding and plastic strain increments. Each increment in plastic strain can be associated with a collective dislocation event, which via dislocation dynamics (DD) simulations have been ascribed to active spiral arm sources as the dominant underlying mechanism [39-41]. One avalanche is thus the rapid activity of one or several sources whose net displacement is recorded as an axial displacement magnitude.

Figure 1(b) displays a force-displacement curve resulting from one loading experiment, highlighting the moments at which SEM images were captured during loading. Between image 1 and 2, we detect the formation of a new slip line, and a second new slip line forms between image 2 and 3 . Although each slip line can trivially be associated with the corresponding large events recorded during compression, a series of smaller events happening in rapid succession may also be detected and must be accounted for. These events may precede or succeed larger events, and their classification is addressed at a later stage in this paper.

Derived from all available deformation data, 817 events were extracted with magnitudes ranging between the experimental displacement resolution limit of $\sim 0.2 \mathrm{~nm}$ and $2551 \mathrm{~nm}$ as the largest detected event magnitude. Out of the 817 events, a total of $516(\sim 63 \%)$ could unambiguously be linked to a pair of pre-event and post-event images. Subsequently, all recorded image pairs were evaluated with respect of the degree of event localization. This means the events were categorized according to if new slip lines were formed, if slip on an already existing slip trace was reactivated, or mixtures of these possibilities. Based on our experimental observation, the data were divided into the following different categories:

\#0: no image pair was captured;

\#1: no resolvable change of the slip morphology could be resolved based on the image pair;

\#2: exactly one new slip line was identified;

\#3: more than one new slip line was identified;

\#4: one existing slip line was found to be reactivated;

\#5: more than one existing slip line was found to be reactivated;

\#6: a combination of at least two traces as per the categories 2-5; 


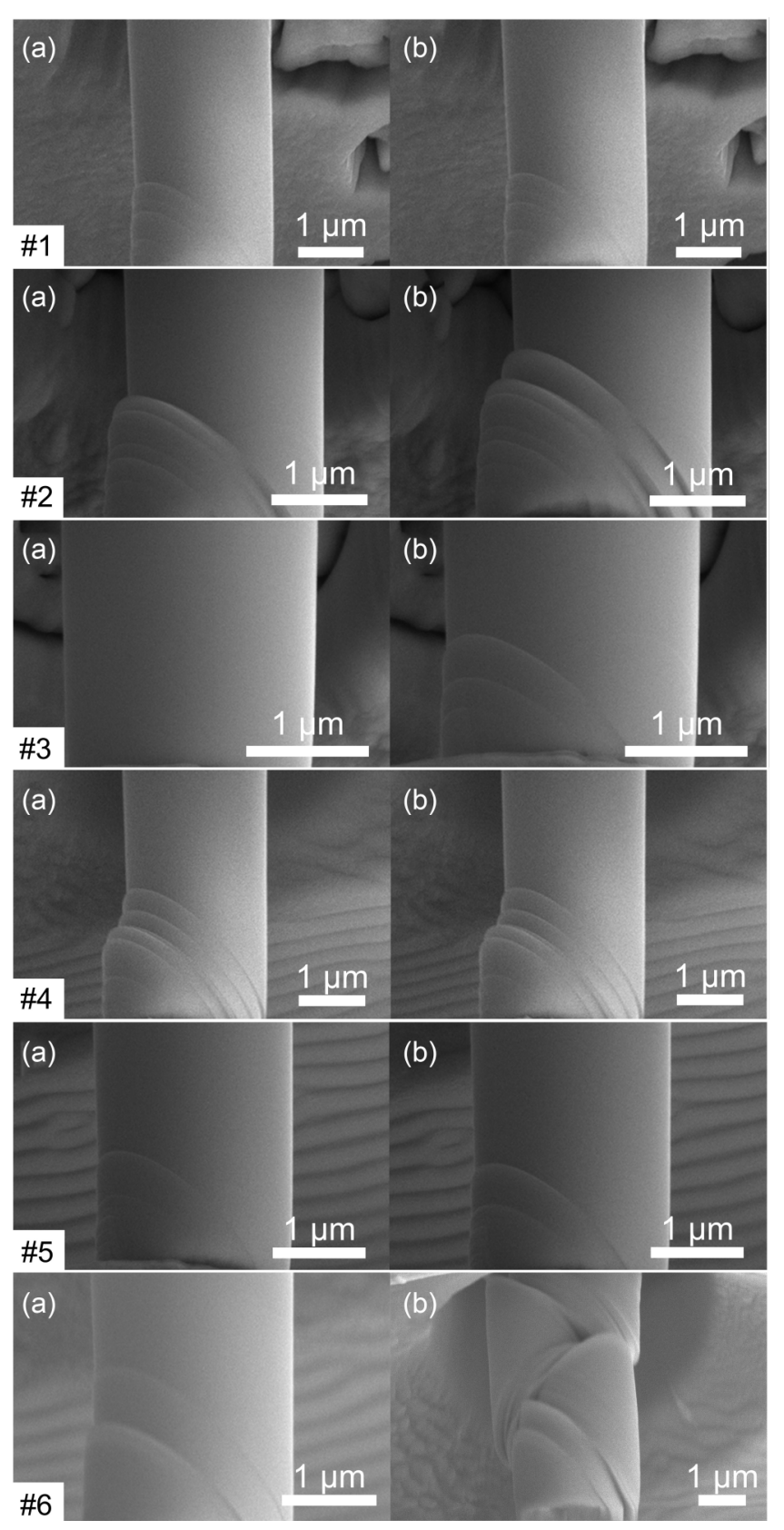

FIG. 2. Categories \#1-\#6 of slip morphology observed during in situ experimentation. For each category, subpanel (a) refers to an image taken before the event was recorded, and subpanel (b) shows the image taken immediately after the event.

\#7: poor image quality or other experimental limitations prevented clear conclusion on the event category, but a mechanical event was captured nonetheless.

Figure 2 displays an example for categories \#1-\#6, omitting \#7, since it collects image data with insufficient quality for an unambiguous analysis. We emphasize that this classification is entirely based on the obtained dataset for the alloy and the experimental resolution. However, to ensure that the classification of slip-morphology images is not biased by the individual who conducted the analysis, we have randomly selected subsets of images that were classified according to categories \#1-\#6 by a different person. No relevant difference in the category assignment of images could be identified. The overall data shows further that no resolvable changes in the slip-line morphology occurred during other stress-strain segments than the events themselves.

Before addressing the statistical analysis of the slip-line morphology pursued here, it is worthwhile to note that the recorded image data does not reveal any indication for a slip morphology that differs from what is seen in the case of pure face-centered cubic (fcc) single crystals. This means, even though HEAs may exhibit enhanced cross-slip and therefore can show signatures of wavy slip in transmission electron microscopy and simulations [42,43], the SEM-based observations do not provide hints of this. This agrees with our earlier work, where the time-resolved velocity profiles of the avalanches were discussed in detail [13]. Indeed, it was found that slip proceeds statistically with the same peak velocity in both pure $\mathrm{Au}$ and the same HEA as studied here, irrespective of slip magnitude. At the coarse-grained level of our experiment, any potential pronounced cross-slip activity and therefore wavy slip in the HEA relative to a pure fcc crystal does thus not manifest itself in the studied avalanche dynamics or the slip-line morphology. Given the rather short-range phenomenon of cross-slip, its absence in the data recorded here of a long-range coupling effect appears reasonable.

Following the above-defined classification scheme, a statistical analysis of the dataset was done. Instead of using a probability density function [PDF, $P(S)]$, we focus on the complementary cumulative distribution function [CCDF, $C(S)$ :

$$
C(S)=P(S \geqslant s)=D \int_{s}^{\infty} P(S) d S,
$$

which denotes the probability for a recorded event size $S$ to be of a magnitude larger than or equal to a given size $s$ and where $D$ is some prefactor. The CCDF has the advantage of being defined for any event size and does not require data binning, unlike the construction of the PDF. We consider that $P(S) \propto S^{-\alpha} e^{-\delta S}$, where $\alpha$ is the scaling exponent, and $e^{-\delta S}$ denotes some cutoff function that is dictated by a nonuniversal numerical value $\delta$. This value may represent a finite-size truncation of the PL or is originating from microstructural length scales that impose correlation-length limitations for the underlying collective dislocation event. Consequently,

$$
C(S) \propto S^{-\tau} e^{-\lambda S},
$$

where $\tau=\alpha-1$, and $\lambda$ is yet another nonuniversal parameter determining the cutoff of the TPL.

Using the MLE method [37] and the POWERLAW Python package [38], we evaluate first the complete dataset shown in Fig. 3 with respect to the statistically most suitable distribution by fitting $P(S)$. When considering a Weibull distribution, an exponential distribution, and a TPL, the latter is found to be the best functional form to capture the full dataset, which is expected for this kind of data where long-range correlated dislocation activity in a finite-sized and plastically flowing crystal is probed. Table I lists the parameters $\alpha$ and $\delta$ for the full dataset and the MLE parameters indicating the suitability of this functional form. These are $R$ (the log likelihood ratio) and $p$ (significance value). In agreement with Refs. [37,44], we consider fitting results with $p<0.05$ as statistically 


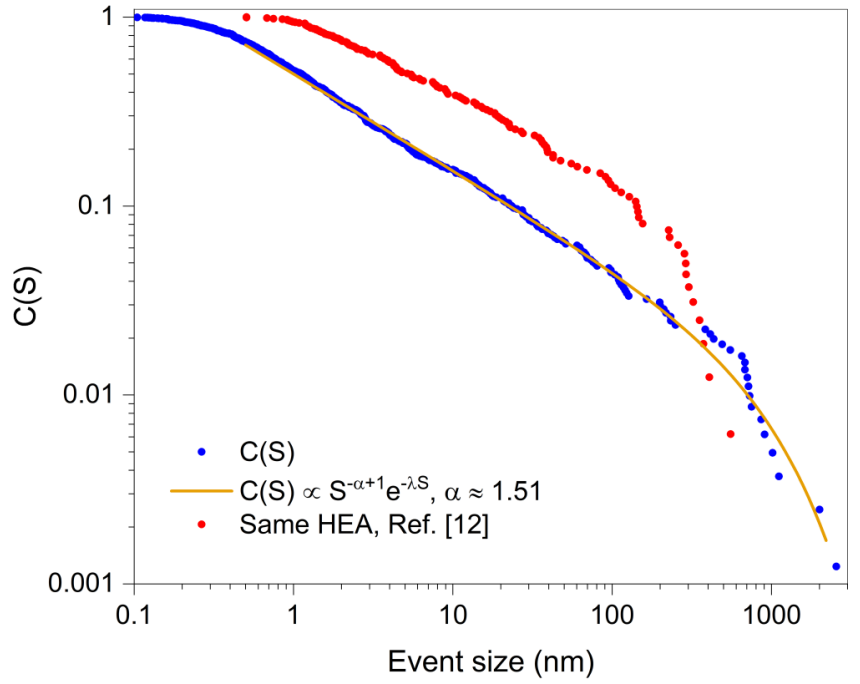

FIG. 3. Cumulative distribution functions (CCDFs) of all measured intermittent events and their truncated power law (TPL) fit. In addition, a much smaller dataset from Ref. [13] is shown for comparison. Despite the different experimental setup and conditions, the exponent and general form obtained when fitting both datasets is very much comparable.

significant. In a comparison between two distributions $A$ and $B$, the first is preferred if $R$ is positive. Table I shows that these criteria for both $R$ and $p$ are fulfilled for a TPL in all cases. The scaling exponent $\alpha$ of the TPL for the entire stress-integrated avalanche-size distribution (Fig. 3) is found to be 1.51, which is smaller than predicted by mean-field approaches, but agrees reasonably very well with the findings in Ref. [13] ( $\alpha=1.27)$ that were based on a total of 162 data points and that also had a slightly higher $x_{\min }$ value, which sets the lower fitting bound of $S$. While the concept of "margin of error" does not apply to estimated values obtained through MLE, we can use the Cramér-Rao bound, which gives the lower bound of the variance of an unbiased estimator (in our case $\alpha$ ). This lower bound is defined by the relationship $e^{2}(\alpha)=\frac{1}{I(\alpha)}$, where $I(\alpha)$ is the Fisher information of the PDF with the value of $\alpha=1.51$ found above. The Fisher information for a (truncated) PL is simply given by $I(\alpha)=\frac{1}{(\alpha-1)^{2}}$. This gives us a variance $e_{\alpha}^{2}=0.2601$, which shows that the previously found $\alpha$ of 1.27 falls within the variance or uncertainty of the scaling exponent obtained here.

Before discussing the occurrence of events of specific categories, it is important to realize that the real-time monitoring

TABLE I. Fit parameters for the two datasets shown in Fig. 3.

\begin{tabular}{lcc}
\hline \hline & This paper & Ref. [13] \\
\hline$\alpha$ for TPL fit & 1.510 & 1.268 \\
$\delta$ for TPL fit & $2.68 \times 10^{-4}$ & $1.9 \times 10^{-3}$ \\
$D_{\text {Ks for TPL fit }}$ & 0.0290 & 0.0455 \\
$\alpha$ for PL fit & 1.551 & 1.479 \\
$R, p$ for TPL vs PL & $3.12 ; 4.2 \times 10^{-3}$ & $3.21 ; 1.89 \times 10^{-5}$ \\
$R, p$ for TPL vs exponential & $11.51 ; 1.12 \times 10^{-30}$ & - \\
$R, p$ for TPL vs Weibull & $2.64 ; 8.26 \times 10^{-3}$ & - \\
\hline \hline
\end{tabular}

during testing is displayed in a window with a reduced datasampling rate at $\sim 60 \mathrm{~Hz}$ in comparison with the $8000 \mathrm{~Hz}$ used to sample and store the data. Because of this discrepancy, it is possible that no event could be seen in the real-time monitoring of the displacement-time data, but that the stored and subsequently analyzed data reveal that an avalanche has taken place. Furthermore, events can be very closely spaced in time, such that it is experimentally impossible to respond with halting the deformation experiment before a new event has occurred. These experimental complications lead to the choice of classifying each of these closely spaced events under the same category. An example of closely spaced events is seen in Fig. 1(b), where some small events are highlighted before the first large displacement jump between image 1 and 2. All the events recorded between both images, such as the large event and the smaller precursor events, are classified as \#2, based on the change in morphology observed between both images. Consequently, the magnitude of the large slip event between image 1 and 2 does include the displacement contribution of the smaller events. A similar situation may arise with small additional avalanches occurring immediately after a larger event. In the case of categories \#2 and \#4, the choice made to bundle these events in the same category is appropriate, since the corresponding image data indeed determined the classification as \#2 or \#4. The choice is less certain when considering categories \#3, \#5, or \#6, where several contributions to the slip-line morphology are observed, but experimental limitations do not allow us in such cases to correlate one event with a single contribution. In other words, what is categorized as a series of \#3 events could be closely spaced \#2 events that could not be resolved individually. However, since most \#3, \#5, and \#6 type events are clearly linked to one forcedisplacement increment, following the same categorization logic used for \#2 and \#4 is deemed a sensible approach. We note that the fraction of such uncertain events amounts to $\sim 8 \%$ for \#3, $22 \%$ for \#5, and $18 \%$ for \#6. Despite these experimental uncertainties, we will now consider the spread and statistical distribution of each of the empirically defined slip categories \#0-\#6.

Figure 4 highlights the individual data-point contribution to the total CCDF, where the largest $10 \%$ of the data for each category is highlighted in green. Events not being classified (category \#0) are clearly favoring smaller avalanches sizes, of which $90 \%$ fall below $3.2 \mathrm{~nm}$ [Fig. 4(a)]. Events smaller than $\sim 0.2 \mathrm{~nm}$ can be disregarded as they are at the limit of the practical displacement resolution. All \#0 events are defined as isolated events that were not immediately resolvable during in situ testing but were later detected during data processing after the actual experiment.

A somewhat similar range of event sizes is covered by category \#1, shown in Fig. 4(b), but the total number of image pairs that do not reveal any resolvable change in slip morphology is much smaller ( $\sim 50$ slip events) than the number of events in \#0. This category simply includes slip events detected during in situ testing, but for which the recorded image pair did not reveal any resolvable difference. This can, for example, occur if the slip event localized outsize of the SEM image bounds, which includes the base of the sample, the view upon which is partly blocked by the surrounding bulk material under the tilted imaging 

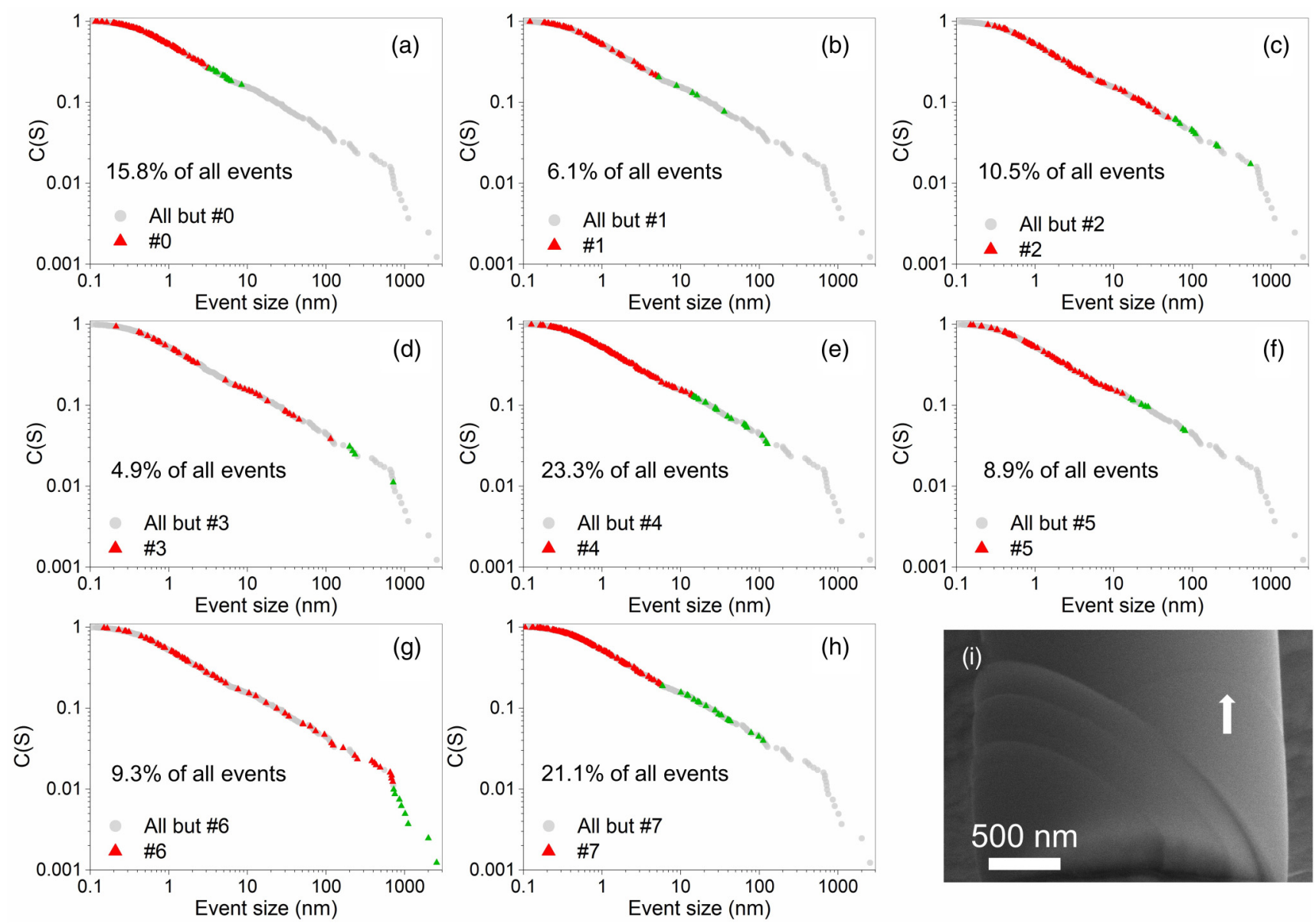

FIG. 4. (a)-(h) Distributions of size range for each event category, with the percentage of the total dataset $(n=817)$ indicated. Data points highlighted in green are the largest $10 \%$ of the event sizes recorded in each category. (i) Example of a scanning electron microscope (SEM) image with a single new slip line (indicated by white arrow) linked to a $3 \mathrm{~nm}$ displacement jump. The other visible slip lines in (i) were assigned to previous events.

conditions. It is also possible that very small increments of already existing slip lines occurred, but in cases of an already complex slip-line morphology, these may not be resolvable unambiguously.

A total of 86 slip avalanches could be ascribed to the formation of exactly one new slip line [\#2, Fig. 4(c)]. These events shift markedly to larger sizes, but also overlap in the 1-10 nm regime with \#0 and \#1. This confirms that the inability to associate a change in the slip-line morphology with an image pair in category \#1 is not due to a limit in experimental slip-line morphology (image) resolution. Indeed, Fig. 4(i) shows a newly formed slip line that caused an axial displacement jump of $\sim 3 \mathrm{~nm}$. As can be seen in the image, such small surface steps and their changes can be reliably captured and traced along the entire deformation curve.

Instead of one new slip line forming during a displacement jump, multiple new slip lines could emerge. These events are classified in category \#3 and shown in Fig. 4(d). They represent the smallest fraction of all categories $(4.9 \%)$. The 40 slip events cover the same size range as category \#2, spanning from approximately 1 to $1000 \mathrm{~nm}$. Category \#4 (reactivation of an already existing slip line, 191 events) is the largest category (most common type of events) and spans up to $\sim 100 \mathrm{~nm}$, with $90 \%$ of the data falling below $10 \mathrm{~nm}$. Thus, the activation of one new slip plane is approximately half as probable as the reactivation of one slip plane, and reactivation yields smaller event sizes if considering the largest $90 \%$ of the data. This suggests that newly created slip lines with so far inactive dislocation sources (\#2) seem to be able to produce a larger number of Burgers vectors that are deposited onto the surface. Based on earlier DD results showing a dominance of single-armed dislocation sources [39-41], this translates into many revolutions before arrest when the local stress has reduced below the required level. The high probability of reactivating exactly one new slip plane (\#4) also indicates that there is no depletion of available sources on already active slip planes.

Category \#5 (reactivation of several slip lines, 73 events) populates a similar size range of up to $100 \mathrm{~nm}$ as \#4. The reactivation of already active slip planes or sources does therefore not seem to affect the number of deposited Burgers vectors. Comparing \#2 with \#3, as well as \#4 with \#5, shows that localization of dislocation activity onto one slip plane instead of multiple is about twice as probable in both cases. This can be understood based on an internal critical stress 
TABLE II. TPL parameters for each subcategory, as well as the original distribution. The $R$ and $p$ values are the result of the comparison between the MLE TPL and Weibull fits. The generally high values of $p$ indicate a statistical ambiguity between both considered functional forms.

\begin{tabular}{lccccc}
\hline \hline Type of event & $\tau$ & $\lambda$ & K-S distance & $R$ & $p$ value \\
\hline \#0: No image & 0.610 & 0.181 & 0.056 & 2.31 & 0.021 \\
\#1: No visible change & 0.593 & 0.0224 & 0.087 & 1.39 & 0.165 \\
\#2: One new slip line & 0.312 & $2.56 \times 10^{-3}$ & 0.061 & 1.52 & 0.128 \\
\#3: Several new slip lines & 0.320 & $1.27 \times 10^{-3}$ & 0.091 & 1.03 & 0.498 \\
\#4: One reactivation & 0.539 & $5.12 \times 10^{-3}$ & 0.053 & 2.31 & 0.843 \\
\#5: Several reactivations & 0.756 & $9.83 \times 10^{-3}$ & 0.078 & 1.18 & 0.021 \\
\#6: Combination & 0.091 & $3.51 \times 10^{-4}$ & 0.102 & 3.76 & 0.239 \\
All events & 0.510 & $2.70 \times 10^{-4}$ & 0.029 & 2.64 & 0.845 \\
\hline \hline
\end{tabular}

distribution for the activation of present dislocation sources, meaning that it is less likely that two closely valued critical stresses populate the low-end tail of this distribution. This, of course, does not consider the possibility of slip activation on another plane while slip already proceeds, which is beyond the experimental resolution.

Category \#6 (complex traces) is overrepresented in the larger size range; indeed, most events ranging between 100 and $2551 \mathrm{~nm}$ in size belong to this category, as shown in Fig. 4(g). A detailed comparison of the values in Table II and the data in Figure 4(g) show us that the tail of the overall distribution is almost exclusively comprised of \#6 events. This is particularly interesting given the limited insight we have about the underlying causes for the truncation of the PL behavior. It has been suggested that the truncation length scale is a result of limiting internal (length scales of dislocation structures or other microstructural features) and external length scales (finite sample size) [18]. However, previous experiments have shown that the introduction of dense dislocation patterns and cell structures within the sample had little to no effect on the observed cutoff magnitude, suggesting that avalanche truncation length scale was not necessarily bound by the intrinsic length scales of the sample [5,25]. Here, we observe that the truncation is linked to avalanches that extend throughout the entire sample, which drives the idea that the truncation is not primarily set by the smallest physical sample dimension either. Instead, this points toward a dominant role of the internal distribution of critical stresses to simultaneously activate the network or dislocation sources (multiple slip) rather than a length scale.

Based on Fig. 4, most of the smallest events (\#0 and \#1, maximum $10 \mathrm{~nm}$ large) can either not be captured or are not resolvable. The formation of new slip lines and reactivation of existing slip lines are of similar size and correspond to $\sim 80 \%$ of the events between 10 and $100 \mathrm{~nm}$, whereas only category \#6 noticeably contributes to the exponential tail of the distribution. These largest events are all of such complex slip morphology that they cannot be further distinguished, and many simultaneously acting and spatially distributed dislocation sources are required to slip more than $\sim 100 \mathrm{~nm}$. The largest event magnitudes are exclusively obtained by the largest degree of avalanche delocalization. Finally, the \#7 category, corresponding to erroneous image data, ranges roughly up to $100 \mathrm{~nm}$ and will not be considered further in the remainder of this paper.
As a next step, we consider the distributions for each category separately to better understand their contribution to the overall event-size distribution. To this end, we isolate the categories defined based on the image data and treat each set separately by again considering MLE fitting. A TPL and the Weibull distribution defined as follows

$$
P(S) \propto k \eta^{k} S^{k-1} e^{-(\eta S)^{k}} \quad \text { for } S \geqslant 0,
$$

which gives us the corresponding $\mathrm{CCDF}$ as

$$
C(S) \propto e^{-(\eta S)^{k}} \quad \text { for } S \geqslant 0,
$$

capture the data best, where the Weibull distribution is described by its shape parameter $k$ and its scale parameter $\eta$. The parameters resulting from the MLE analysis for each category, as well as the original distribution, are summarized in Table II. The table also includes the Kolmogorov-Smirnov (K-S) distance, which is the preferred goodness-of-fit test in the context of MLE fitting: it compares the empirically measured distribution function with the reference distribution determined by MLE and outputs the maximum distance between both distributions. An expectation is that the subcategories cannot be of TPL type, since a sum of PLs does not yield a PL and given the high $p$ values in Table II, it was not possible to conclude which of the two distribution functions best describe the data.

However, this approach reinforces the conclusions that can be made from Fig. 4, namely, that a subset of the overall distribution has its specific size range. When graphing the data of Table II in Fig. 5, two overall sets emerge. Indeed, all slip categories involving dislocation avalanches that contain reactivation of already active slip planes decay faster and strongly contribute to the smaller avalanche magnitudes, whereas avalanches that also proceed on newly formed slip planes have a shallower decay and larger magnitudes.

In addition to focusing on the event sizes (statics), it is further possible to resolve the time-dependent velocity profile of the occurring dislocation avalanches. This has been demonstrated in a series of earlier efforts that either reveal good agreement with mean-field models [22] or reveal clear microstructural dependencies of peak velocity, velocity relaxation, and scaling behavior [12,27,45]. Since the velocity size scaling of the data acquired here has a numerically identical 


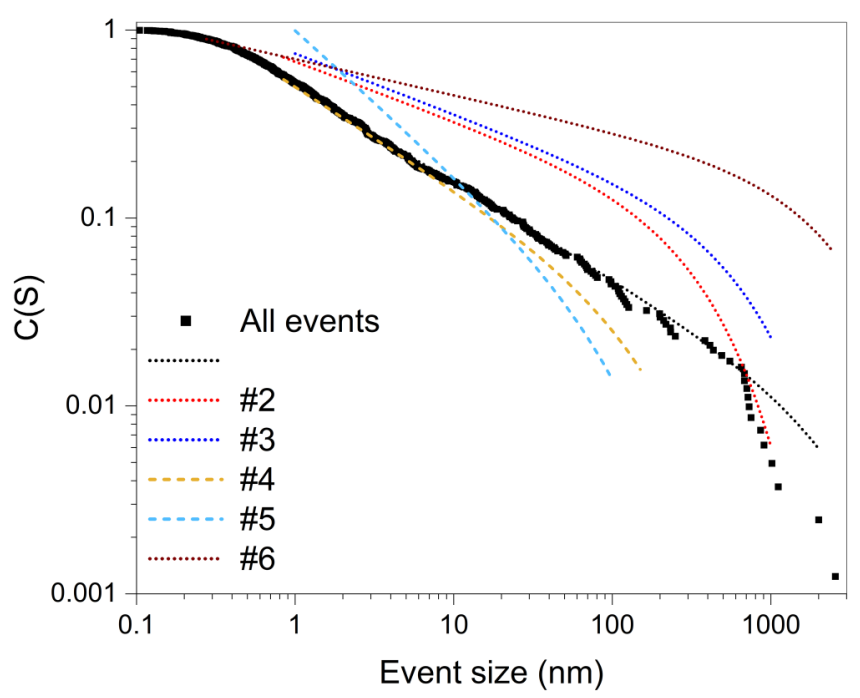

FIG. 5. Cumulative distribution functions (CCDFs) of subcategories \#2-\#6, using the fitting results listed in Table II. Each fit is only defined for the relevant size range of its category. Categories \#2, \#3, and \#6, involving newly activated slip planes, distribute shallower than the subsets that only involve reactivation of existing slip lines.

scaling exponent, as reported in Ref. [13], we will limit ourselves to the comparison between the categories that involve new slip-line formation (\#2, \#3, and \#6) and those that only involve reactivation (\#4 and \#5). In a simple histogram representation shown in Fig. 6, the peak velocity of avalanches involving new slip-line formation is distinctly populating the higher velocity tail of the distribution. Indeed, events that involve the formation of new slip lines can reach peak velocities that are up to one order of magnitude higher than when reactivation of already activated slip planes occurs.

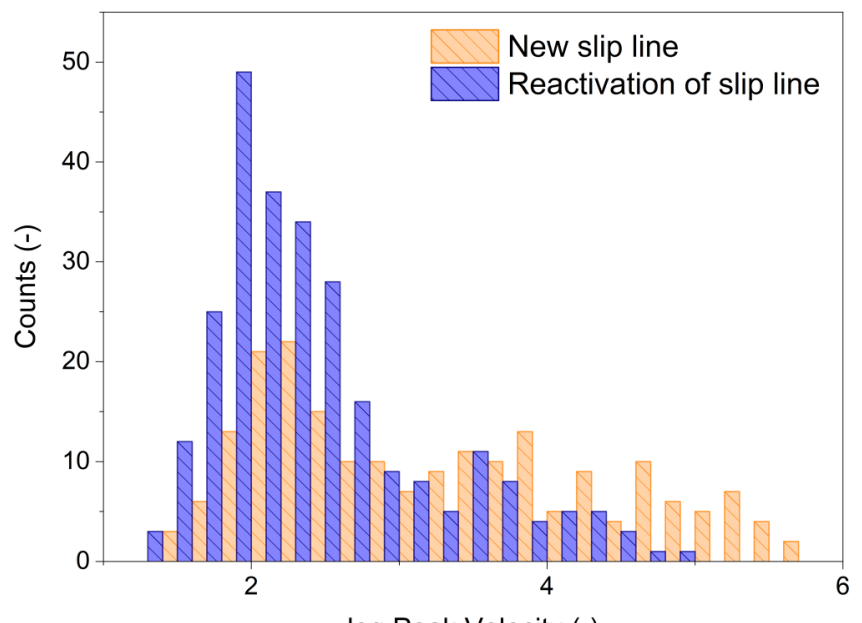

$\log$ Peak Velocity (-)

FIG. 6. Histogram of log value of recorded peak velocities summarized in two datasets for events corresponding to new slip lines or the reactivation of an existing slip line, respectively. While both datasets show a peak in the range $\log \left(v_{\text {peak }}\right) \approx 1.8-2.6 \rightarrow v_{\text {peak }} \approx$ $60-400 \mathrm{~nm} \mathrm{~s}^{-1}$, the peak velocities involving the formation of new slip lines clearly populated higher velocities.
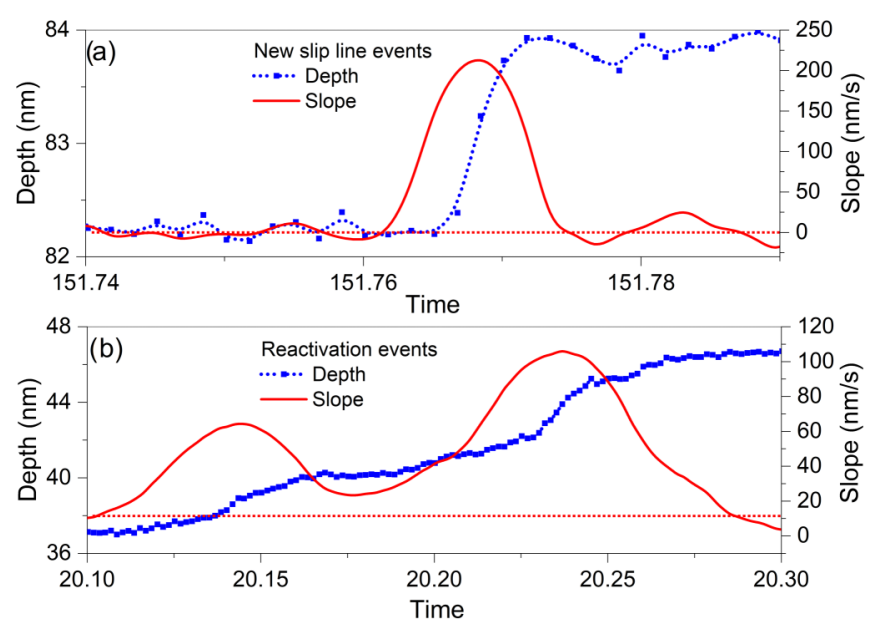

FIG. 7. Displacement-time and velocity-time trace for (a) an avalanche leading to new slip lines, and (b) an avalanche involving the reactivation of an already existing slip line. Events leading to a new slip line show sharp velocity profiles, whereas events involving the reactivation of slip lines have an in-time extended fluctuating velocity trace.

This difference in avalanche velocities can be reconciled through a detailed examination of the displacement-time traces. Overall, it becomes clear that the events belonging to the new slip-line type exhibit sharp and distinct velocity profiles that are composed of one short acceleration-deceleration cycle, whereas the events of reactivation type often display more irregular and in-time dragged out velocity profiles with several acceleration-deceleration cycles. Figure 7 displays two such cases, where in Figure 7(a) the fast and sharp events of two newly formed slip lines is shown, and Fig. 7(b) depicts the reactivation type that proceeds slowly and reaches a lower peak velocity. This suggests that the reactivation event type is composed of a sequence of subevents that are not individually resolvable in the experiments but are sufficiently separated in time such that the displacement accumulation occurs over a longer time and at slower velocity. This observation sheds some light onto earlier findings of different event types that were not understood [46]. We finally note that this feature of more irregular and in-time extended velocity profiles of the reaction type is not a sharp discriminator, but a clear tendency is seen in the data. As such, the higher peak velocity range in Fig. 6 can be understood, but it should be clear that these findings are related to statistical trends. In numbers, this means that $\sim 50 \%$ of all events forming new slip lines are composed of one $(\sim 35 \%)$ or two $(\sim 15 \%)$ resolvable subpeaks, whereas $50 \%$ of the events of reactivation type are distributed over $1-5$ subevents, with only $10 \%$ featuring one isolated velocity profile.

\section{CONCLUSIONS}

In summary, we have traced the morphological signature of microplastic slip in a prototypical HEA during deformation, where the overall dataset of slip-size increments follows TPL scaling with a nontrivial scaling exponent. This scale-free distribution itself is remarkable, as it demonstrates that the presumably spatially complex dislocation mobility law of the 
HEA does not seem to alter the universally observed scale-free scaling that emerges due to long-range physics. Our results allow us to link a given event size of a dislocation avalanche with the resulting slip-line pattern - that is the degree of spatial avalanche localization on the surface of the deforming sample. Based on the morphological observations, categories of slip-line patterns were defined, of which the analyzable categories either represent dislocation avalanches that occur on already activated slip planes, or forming new slip lines. Both reactivation and the formation of new slip lines onto exactly one slip line is approximately twice as likely as mechanisms occurring in a more delocalized manner on several slip lines. A high degree of delocalized avalanching involving numerous slip lines is always a mixture of reactivation and the formation of new slip lines. These delocalized events stand out, as they essentially represent the exponential tail of the TPL scaling and therefore set the truncation length scale of the distribution. A more detailed analysis of the experimentally defined subcategories of slip-line morphology shows that avalanches involving new slip-line formation are statistically larger in size and therefore tend to contribute to a reduction in the scaling exponent. The opposite is seen for avalanches involving only reactivation of slip lines. Furthermore, a statistical tendency of faster avalanches is seen when involving new slip-line formation in comparison with pure reactivation. The corresponding time-resolved velocity profiles show that faster events of newly activated slip planes tend to be singular and sharp, whereas the slow events of pure reactivation type are found to be extended in time and with resolvable timedependent velocity oscillations.

These observations shed light onto how different morphological and spatial signatures of dislocation avalanches contribute to the overall scaling behavior of the event sizes in intermittent microplasticity. A spectrum of classifiable microstructural signatures is observed that underlies the observed scale-free behavior. While the physics perspective often focuses on the overall scaling exponent and the possible universality class (or classes), the link established here between the statistics and slip morphologies motivates the more materials science approach of focusing on the underlying microstructural changes, giving insight into the geometry and dimensionality of the avalanches, all of which ultimately will give insight into the origin of the nontrivial exponents.

\section{ACKNOWLEDGMENTS}

The authors thank G. Sparks for introducing Q.R. to the analysis code. This research was carried out in part at the Frederick Seitz Materials Research Laboratory Central Research Facilities, University of Illinois. R.M. is grateful for financial support by the NSF CAREER Program (Grant No. NSF DMR 1654065) and for startup funds provided by the Department of Materials Science and Engineering at UIUC.
[1] S. R. Macewen and B. Ramaswami, Philos. Mag. 22, 1025 (1970).

[2] B. J. Brindley and J. T. Barnby, Acta Metall. 14, 1765 (1966).

[3] R. Maaß, D. Klaumünzer, and J. F. Löffler, Acta Mater. 59, 3205 (2011).

[4] R. Maaß and J. F. Löffler, Adv. Funct. Mater. 25, 2353 (2015).

[5] R. Maaß and P. M. Derlet, Acta Mater. 143, 338 (2018).

[6] E. Orowan, Processings of the Physical Society 52, 8 (1940).

[7] D. M. Dimiduk, C. Woodward, R. LeSar, and M. D. Uchic, Science 312, 1188 (2006).

[8] M. C. Miguel, A. Vespignani, S. Zapperi, J. Weiss, and J. R. Grasso, Nature 410, 667 (2001).

[9] M. Zaiser, Adv. Phys. 55, 185 (2007).

[10] J. Weiss and J. R. Grasso, J. Phys. Chem. B 101, 6113 (1997).

[11] T. Richeton, P. Dobron, F. Chmelik, J. Weiss, and F. Louchet, Mater. Sci. Eng. A 424, 190 (2006).

[12] G. Sparks and R. Maaß, Acta Mater. 152, 86 (2018).

[13] Q. Rizzardi, G. Sparks, and R. Maaß, JOM 70, 1088 (2018).

[14] J. Shimanek, Q. Rizzardi, G. Sparks, P. M. Derlet, and R. Maaß, J. Mater. Res. 35, 196 (2020).

[15] K. Chatterjee, A. J. Beaudoin, D. C. Pagan, P. A. Shade, H. T. Philipp, M. W. Tate, S. M. Gruner, P. Kenesei, and J. S. Park, Struct. Dyn. 6, 014501 (2019).

[16] A. I. Hegyi, P. D. Ispanovity, M. Knapek, D. Tuzes, K. Mathis, F. Chmelik, Z. Dankhazi, G. Varga, and I. Groma, Microsc. Microanal. 23, 1076 (2017).

[17] M. Zaiser, J. Schwerdtfeger, A. S. Schneider, C. P. Frick, B. G. Clark, P. A. Gruber, and E. Arzt, Philos. Mag. 88, 3861 (2008).
[18] F. F. Csikor, C. Motz, D. Weygand, M. Zaiser, and S. Zapperi, Science 318, 251 (2007).

[19] S. Brinckmann, J. Y. Kim, and J. R. Greer, Phys. Rev. Lett. 100, 155502 (2008).

[20] J. R. Greer, W. C. Oliver, and W. D. Nix, Acta Mater. 53, 1821 (2005).

[21] A. A. Shibkov, M. F. Gasanov, M. A. Zheltov, A. E. Zolotov, and V. I. Ivolgin, Int. J. Plast. 86, 37 (2016).

[22] M. LeBlanc, L. Angheluta, K. Dahmen, and N. Goldenfeld, Phys. Rev. E 87, 022126 (2013).

[23] J. T. Uhl et al., Sci. Rep. 5, 16493 (2015).

[24] P. D. Ispanovity, I. Groma, G. Gyorgyi, F. F. Csikor, and D. Weygand, Phys. Rev. Lett. 105, 085503 (2010).

[25] G. Sparks and R. Maaß, Phys. Rev. Materials 2, 120601(R) (2018).

[26] S. Papanikolaou, D. M. Dimiduk, W. Choi, J. P. Sethna, M. D. Uchic, C. Woodward, and S. Zapperi, Nature 490, 517 (2012).

[27] G. Sparks and R. Maaß, Eur. Phys. J. B 92, 15 (2019).

[28] H. Salmenjoki, A. Lehtinen, L. Laurson, and M. J. Alava, Phys. Rev. Materials 4, 083602 (2020).

[29] P. Zhang, O. U. Salman, J. Weiss, and L. Truskinovsky, Phys. Rev. E 102, 023006 (2020).

[30] G. Sparks, P. S. Phani, U. Hangen, and R. Maaß, Acta Mater. 122, 109 (2017).

[31] M. Zaiser, K. Bay, and P. Hahner, Acta Mater. 47, 2463 (1999).

[32] P. Hahner, K. Bay, and M. Zaiser, Phys. Rev. Lett. 81, 2470 (1998).

[33] G. Sparks, Y. Cui, G. Po, Q. Rizzardi, J. Marian, and R. Maaß, Phys. Rev. Materials 3, 080601(R) (2019). 
[34] D. E. Kramer, M. F. Savage, and L. E. Levine, Acta Mater. 53, 4655 (2005).

[35] M. Zaiser, F. M. Grasset, V. Koutsos, and E. C. Aifantis, Phys. Rev. Lett. 93, 195507 (2004).

[36] W.-R. Wang, W.-L. Wang, S.-C. Wang, Y.-C. Tsai, C.-H. Lai, and J.-W. Yeh, Intermetallics 26, 44 (2012).

[37] A. Clauset, C. R. Shalizi, and M. E. J. Newman, Siam Review 51, 661 (2009).

[38] J. Alstott, E. Bullmore, and D. Plenz, PLoS ONE 9, e85777 (2014).

[39] S. I. Rao, D. M. Dimiduk, M. Tang, M. D. Uchic, T. A. Parthasarathy, and C. Woodward, Philos. Mag. 87, 4777 (2007).
[40] S. I. Rao, D. M. Dimiduk, T. A. Parthasarathy, M. D. Uchic, M. Tang, and C. Woodward, Acta Mater. 56, 3245 (2008).

[41] X. H. Liu and K. W. Schwarz, Model. Simul. Mater. Sci. Eng. 13, 1233 (2005).

[42] E. Ma, Scr. Mater. 181, 127 (2020).

[43] Z. Wang, I. Baker, Z. Cai, S. Chen, J. D. Poplawsky, and W. Guo, Acta Mater. 120, 228 (2016).

[44] A. Chittaranjan, Indian J. Psychol. Med. 41, 210 (2019).

[45] A. Dobrinevski, P. Le Doussal, and K. J. Wiese, EPL (Europhysics Letters) 108, 66002 (2014).

[46] R. Maaß, P. M. Derlet, and J. R. Greer, Small 11, 341 (2015). 